\title{
A Technique of Bilateral Inguinal Hernia Repair Using 10 mm Single Port Access and Bioresorbable Composite Mesh Fixed with Endoclose Sutures: Three Cases Reported
}

\author{
Wuttichai Thanapongsathorn \\ HRH Princess Maha Chakri Sirindhorn Medical Center, Department of Surgery, Faculty of Medicine, \\ Srinakharinwirot University, Nakron-Nayok, Thailand \\ E-mail:wutticha@hotmail.com \\ Received April 22, 2011; revised May 3, 2011; accepted May 24, 2011
}

\begin{abstract}
Purpose: To report a novel technique of laparoscopic $10 \mathrm{~mm}$ Single Port Access IntraPeritoneal Onlay Mesh (SPA-IPOM) using bioresorbable composite mesh fixed with Endoclose suture (percutaneous subcutaneous suture) in 3 cases of bilateral inguinal hernia. Methods: Laparoscopic SPA-IPOM is done through a $10 \mathrm{~mm}$ trocar with one $10 \mathrm{~mm}$ instrument that has $5 \mathrm{~mm}$ optical lens and $5 \mathrm{~mm}$ channel for grasper. After inserting $10 \mathrm{~mm}$ trocar at umbilicus using semi-open technique, intraperitoneal anatomical landmark of inguinal hernia is identified. A $10 \times 15 \mathrm{~cm}$ pre-tied bioresorbable composite mesh is then placed to cover hernia defect and all three potential area of indirect, direct and femoral hernia. Using Endoclose ${ }^{\mathrm{TM}}$ needle, each pair of pre-tied sutures is retrieved percutaneously through a needle wound and extracorporeal tied with knot in subcutaneous space. After the upper half of mesh is sutured to the posterior surface of abdominal wall, the lower half of mesh is fixed by hernia tacker to Symphysis Pubis, Cooper Ligament and Iliopubic tract. Results: Three men, average 48 year olds were operated by laparoscopic $10 \mathrm{~mm}$ SPA-IPOM for bilateral inguinal hernia repair using bioresorbable composite mesh. Average operative time was 36 minutes. No immediate complication. All patients were discharge on the $2^{\text {nd }}$ post-operative day and average 6 months follow up has no recurrence. Conclusions: Laparoscopic SPA-IPOM is an optional operation and is much easier to be performed. Benefits include operative time saving, cosmesis, early discharge and early return to work. Bioresorbable composite mesh prevents bowel adhesion, however, is much more expensive. Long term follow up study for complications and recurrence is needed.
\end{abstract}

Keywords: 10 mm Single Port Access, IntraPeritoneal Onlay Mesh, Percutaneous Subcutaneous Suture, Bioresorbable Composite Mesh, Bilateral Inguinal Hernia Repair

\section{Introduction}

Hernias occur fairly frequently and are more common in adult males. Occasionally these can be life threatening or more frequently simply a painful nuisance. Nonetheless, the only true remedy is surgical repair. Over centuries surgical techniques have evolved until the present whereby laparoscopic approaches predominate. Several various methodologies have been introduced [1]. We now wish to add to this growing laparoscopic experience and offer three cases of bilateral inguinal hernia repair. To our knowledge, we are the first to report on utilizing a $10 \mathrm{~mm}$ single port access intraperitoneal onlay mesh [SPA-IPOM] incorporating bioresorbable composite mesh fixed with Endoclose suture (percutaneous subcutaneous suture) in bilateral inguinal hernia repair.

\section{Cases Report}

A total of three Thai men average age 48 years old [range 32 - 58] presented with symptomatic bilateral inguinal hernias. One was a bilateral indirect and the remaining 2 bilateral direct inguinal hernias. All underwent repair using the laparoscopic technique described in the next section after informed consent was obtained.

The average operative time was 36 minutes [range 28 50] without any immediate complications. The patients have to be admitted due to health insurance system of the 
hospital and were all discharged on the $2^{\text {nd }}$ post-operative day. Follow up one week later failed to reveal seroma, wound infection, nor evidence of gut obstruction, fistula or pain from mesh related complication. To date [6 months] no recurrence has been noted in any of the patients and all enjoyed an excellent cosmetic result. (Figure 1)

\section{Operative Technique}

Laparoscopic SPA-IPOM was performed via a $10 \mathrm{~mm}$ trocar with the following standard set of instruments (Figure 2); A $10 \mathrm{~mm}$ gynecological instrument that has $5 \mathrm{~mm}$ optical lens and $5 \mathrm{~mm}$ channel for grasper, A $43 \mathrm{~cm}$ length of $5 \mathrm{~mm}$ grasper and An Endoclose ${ }^{\mathrm{TM}}$ needle.

Procedure is done under general anesthesia in the following sequence;

1) Inserting a standard $10 \mathrm{~mm}$ trocar at umbilicus using semi-open technique.

2) Intraperitoneal anatomical landmarks involved in inguinal hernia repair were identified; Triangle of Doom or Square of Doom, Symphysis pubis, Cooper ligament, Iliopubic tract and Anterior superior iliac spine. There was no dissection of the peritoneum.

3) Each hernia opening was strengthened with a $10 \times$ $15 \mathrm{~cm}$ pre-tied bioresorbable composite mesh (Parietex ${ }^{\mathrm{TM}}$ composite mesh). (Figure 3) Preparation of the mesh involved placing three $2 / 0$ vicryl sutures $\sim 1 \mathrm{~cm}$ from the superior edge. After wetting for 30 seconds with sterile water, the mesh was rolled in cigarette fashion enabling passage through the $10 \mathrm{~mm}$ port into the peritoneal space. Unrolling the mesh allowed coverage of all 3 potential inguinal hernia areas (indirect, direct and femoral hernia). This form of mesh is two-sided with a bioresorbable collagen side to preclude visceral organ adhesion and another side to promote tissue in-growth for enhanced fixation against posterior abdominal wall.

4) The upper half of mesh is fixed with percutaneous subcutaneous suture at the level of $2 \mathrm{~cm}$ above transverses abdominis arch, medially at abdominal midline, laterally near anterior superior iliac spine and the middle at between the medial and lateral suture. The Endoclose $^{\mathrm{TM}}$ needle pass through abdominal wall into peritoneal cavity under direct vision, grasp and pull out one end of vicryl pre-tied suture outside abdominal wall, then insert Endoclose ${ }^{\mathrm{TM}}$ needle through previous needle wound, grasp and pull out another end of vicryl pre-tied suture, extracorporeal tied with knot in subcutaneous space (Figure 4). So, the upper half of mesh is fixed to posterior abdominal wall firmly.

5) Fixation of the lower half of the mesh was done by using hernia tacker at Symphysis pubis (medial side), Cooper ligament (lower side), Iliopubic tract (lateral

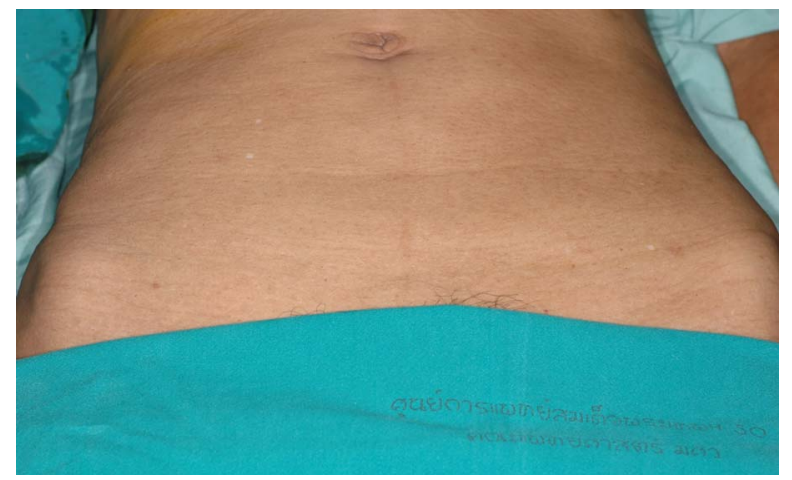

Figure 1. Postopertive cosmetic result.

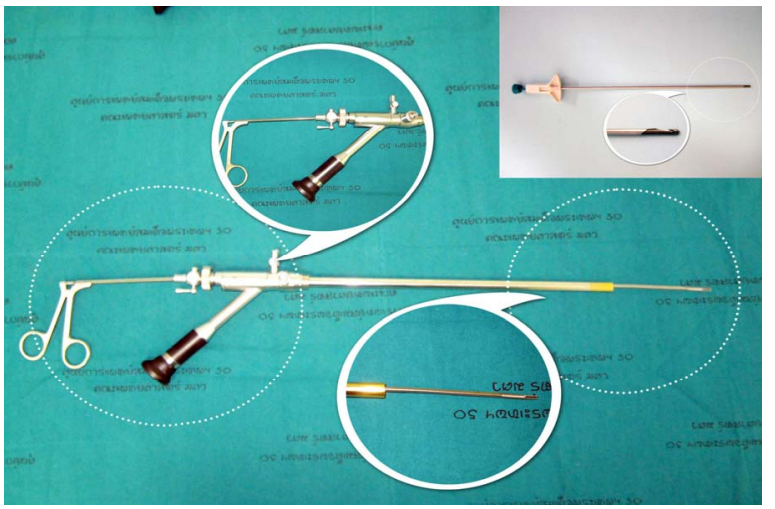

Figure $2.10 \mathrm{~mm}$ gynecological instrument and Endoclose ${ }^{\mathrm{TM}}$ needle.

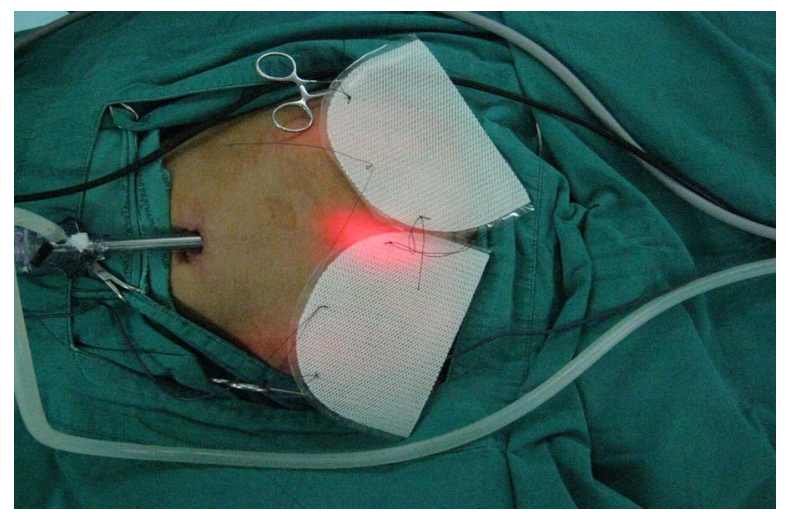

Figure 3. Pre-tied bioresorbable composite mesh.

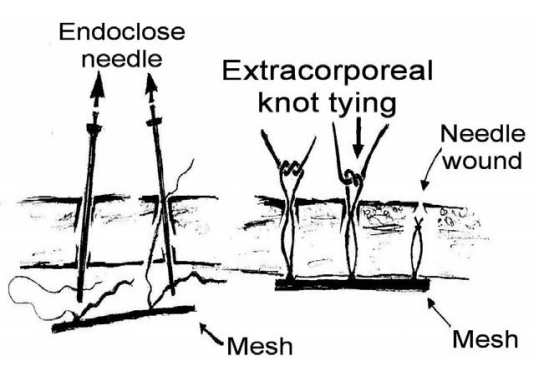

Figure 4. Endoclose ${ }^{\mathrm{TM}}$ needle and extracorporeal knot tying. 
side). Care was taken not to place hernia tacker at Triangle of Doom or Square of Doom.

6) Remove trocar and closure of umbilical wound. Dress needle wounds without suture.

\section{Discussion}

By definition a hernia is a protrusion of an internal organ through a tear, hole or defect in the wall of a body cavity. Most often it is the abdominal wall which is defective and hernias are classified anatomically with inguinal hernias being the most prevalent. Groin hernias were depicted as far back as 1552 BC in ancient Egyptian writing and there are even some findings in mummies that surgery was attempted. For many centuries the anatomic detailing greatly improved but concrete and feasible surgical technique lagged. Despite these important advances in the knowledge of anatomy and the introduction of anesthesia in 1846, surgery on hernias made little progress during the first half of the nineteenth century, as any attempt to open the inguinal canal was followed by severe sepsis and had a near $100 \%$ recurrence rate of the hernia.

Traditional surgical technique used for decades did an open approach with basic simple suture closure of the anatomic defect without addressing or reinforcing the surrounding thinned and weakened hernia-prone tissue layer. These older methods of repair however were associated with significant tension leading to poor wound healing, suture line disruption and hernia recurrence. This paved the way for the development of mesh repair in a truly tension-free fashion as popularized by Lichenstein.

Minimally invasive surgery or Keyhole surgery utilizing laparoscopy is a procedure conceptualized to provide effective standard surgical treatment inside the human body yet decreasing access-related morbidity to abdominal wall. The potential advantages that have been reported are less postoperative pain, decrease blood loss, quicker recovery, superior cosmetic results, less surgical trauma to unrelated organ and surrounding tissue with decreased immunological and metabolic trauma to the patient. In the short $\sim 20$ year history of laparoscopic surgery we have seen an increased worldwide acceptance and progression from multi-port (4 incisions) to single port (one incision). Concurrent with this trend has been improvement in equipment allowing for smaller sized entry sites ranging from $25 \mathrm{~mm}$ to $2 \mathrm{~mm}$ port and placing the incision wound into anatomic hidden areas for improved cosmetic results. Thus leading to the terms "hidden scar" or "scar less surgery".

Laparoscopic hernia surgery has many subtle variations usually reflective of the technique, anatomic approach, materials and means of fixation. A thorough comparative review is beyond the scope of this article but will be roughly outlined. Laparoscopic inguinal hernia repair technique has 3 main modifications in approach; TAPP (Trans Abdominal PrePeritoneal repair) TEP (Totally ExtraPeritoneal repair) and IPOM (IntraPeritoneal Onlay Mesh repair). The type of approach selected by surgeons is based on personal preference and individual skills. For minimizing surgical trauma to abdominal wall and cosmetics, some surgeon use a single incision to perform laparoscopic inguinal hernia repair by inserting either three separate $5 \mathrm{~mm}$ trocars through one skin incision or one special trocar that has $3-4$ channels. There are favorable published reports for both laparoscopic Single Port Access Trans Abdominal PrePeritoneal (SPA-TAPP) inguinal hernia repair [2-4] and laparoscopic Single Port Access Totally ExtraPeritoneal (SPA-TEP) inguinal hernia repair [5-7]. SPA has been reported successful in many various laparoscopic procedures;

- SPA-laparoscopic cholecystectomy [8]

- SPA-laparoscopic appendectomy [9]

- SPA-laparoscopic nephrectomy [10]

- SPA-laparoscopic prostatectomy [11]

- SPA-laparoscopic sigmoidectomy [12]

- SPA-laparoscopic placement of an adjustable gastric band [13]

As mentioned the trend has been from multiple ports to single port laparoscopic surgery. Single port surgery is known by a multitude of names with associated acronyms. Let us clarify some further terminology for discussion;

- Single Incision Laparoscopic Surgery (SILS)

- Single Access Laparoscopic Surgery (SALS)

- Single Access Surgery (SAS)

- One Port Umbilical Surgery (OPUS)

- Single Port Incisionless Conventional Equipmentutilizing Surgery (SPICES)

- Natural Orifice TransUmbilical Surgery (NOTUS)

- Embryonic Natural Orifice Transluminal Endoscopic Surgery (E-NOTES)

- LaparoEndoscopic Single-site Surgery(LESS)

All of these incorporate a single incision $(10-25 \mathrm{~mm})$ usually at the umbilicus minimizing the number of incisions and improved cosmetic results. There are three approaches;

1) One standard $10 \mathrm{~mm}$ port with one $10 \mathrm{~mm}$ instrument that has $5 \mathrm{~mm}$ optical lens and $5 \mathrm{~mm}$ channel for instrument. (10 mm SPA).

2) One special 15 - $25 \mathrm{~mm}$ port that has 3 - 4 channels for $5-10 \mathrm{~mm}$ telescope and hand instruments [14]. (Special SPA: -TriPort ${ }^{\circledR}$, R-port ${ }^{\circledR}$, Endocone $^{\circledR}$, X-cone $^{\circledR}$, SILS port ${ }^{\circledR}$, Uni-X single port ${ }^{\circledR}$, GelPort ${ }^{\circledR}$, SITRACC port $\left.^{\circledR}\right)$. 
3) Two or three $5 \mathrm{~mm}$ port into single incision. One port for $5 \mathrm{~mm}$ telescope, others port for $5 \mathrm{~mm}$ hand instruments. (SI-MPA: Single Incision-Multiple Ports Access).

All three approaches are single incision that will give better cosmetic results than multiple incision procedure. However, specialized trocars are very expensive, require more special hand instruments and demand higher skills and trainings to perform the operation. Our technique of $10 \mathrm{~mm}$ SPA-IPOM using a standard trocar and gynecological instruments which are present in most operating rooms can be performed by those surgeons who possess standard laparoscopic surgical skills.

There are many reports using bioresorbable composite mesh for standard incisional hernia repair [15]. Parietex $^{\mathrm{TM}}$ Composite Mesh was introduced in 1999 with a resorbable collagen barrier on one side to limit visceral adhesion and a three-dimensional polyester knit structure on the other to promote tissue in growth.

The assurance of mesh fixation is still a problem for some surgeons. Olmi in 2007 [16] reported on laparoscopic repair of inguinal hernia using an intraperitoneal onlay mesh (IPOM) technique fixed with fibrin glue (Tissucol). To combat this potential problem we strengthened the mesh fixation both by suturing to the abdominal wall and utilizing tacker to the bony and fascia parts (Symphysis pubis, Cooper ligament, Iliopubic tract). This assured that the mesh will not displace during postoperative activity.

Granted, that any bioresorbable composite mesh is more expensive 3 - 5 times than prolene mesh. One must consider overall benefit to the patient and more efficient hospitalization costs. Laparoscopic repair incorporates all or some of the following advantages:

1) less postoperative pain

2) decrease blood loss

3) less surgical trauma to unrelated organ and surrounding tissue

4) decrease immunological and metabolic trauma to the patient

5) strengthen all three potential inguinal hernia in same procedure (prophylactic role)

6) operative time saving

7) superior cosmetic wound

8) quicker recovery and early discharge

9) early return to work

To date no report has utilized suture for mesh fixation under one $10 \mathrm{~mm}$ trocar SPA-IPOM technique. Our report shows personal technique using $10 \mathrm{~mm}$-Single-Port Access IntraPeritoneal Onlay Mesh (SPA-IPOM) technique with bioresorbable composite mesh (Parietex $^{\mathrm{TM}}$ composite mesh) fixed in place with suture and hernia tacker.

\section{Summary}

Our experience showed that 10mm SPA-IPOM for bilateral inguinal hernia repair fixed with suture and tacker, is an optional operation and easier to perform than TAPP or TEP technique. But prospective randomized controlled trial should be done for long term benefit and cost effectiveness to the patient and health care system. In our approach of Laparo-Endoscopic Single Site Surgery [LESS] and Mesh Onlay Reinforced with Endoclose suture [MORE] perhaps the architects of modern surgery will find like Ludwig Mies van der Rohe that "less is more".

\section{Author's Declaration}

\section{Open Access}

This article is distributed under the terms of the Creative Commons Attribution Noncommercial License which permits any noncommercial use, distribution and reproduction in any medium, provided the original author and source are credited.

\section{References}

[1] C. J. Davis and M. E. Arregui, "Laparoscopic Repair for Groin Hernias,” Surgical Clinics of North America, Vol. 83, December 2003, pp. 1143-1161.

[2] P. Roy and A. De, "Single-Incision Laparoscopic TAPP Mesh Hernioplasty Using Conventional Instruments: An Evolving Technique,” Langenbeck's Archives of Surgery, Vol. 395, No. 8, November 2010, pp. 1157-1160. doi:10.1007/s00423-010-0656-8

[3] E. R. MacDonald and I. Ahmed, “'Scar Less’ Laparoscopic TAPP Inguinal Hernia Repair Using a Single Port,” The Surgeon, Vol. 8, No. 3, June 2010, pp. 179- 181. doi:10.1016/j.surge.2009.11.006

[4] C. Menenakos, M. Kilian and J. Hartmann, "Single-Port Access in Laparoscopic Bilateral Inguinal Hernia Repair: First Clinical Report of a Novel Technique,” Hernia, Vol. 14, No. 3, June 2010, pp. 309-312.

[5] K. He, H. Chen, R. Ding, R. Hua and Q. Yao, "Single Incision Laparoscopic Totally Extraperitoneal Inguinal Hernia Repair,” Hernia, Vol. 15, No. 4, May 2010, pp. 451-453.

[6] O. Surgit, "Single-Incision Laparoscopic Surgery for Total Extraperitoneal Repair of Inguinal Hernias in 23 Patients,” Surgical Laparoscopy, Endoscopy \& Percutaneous Techniques, Vol. 20, No. 2, April 2010, pp. 114-118. doi:10.1097/SLE.0b013e3181d848c3

[7] S. Agrawal, A. Shaw and Y. Soon, "Single-Port LaparoScopic Totally Extraperitoneal Inguinal Hernia Repair with the Triport System: Initial Experience," Surgical Endoscopy, Vol. 24, No. 4, April 2010, pp. 952-956. doi:10.1007/s00464-009-0663-4

[8] H. Mofid and C. Zornig, "Single-Access Surgery Laparo- 
scopic Cholecystectomy and Appendectomy,” Surgical Technology International, Vol. 19, April 2010, pp. 61-64. http://www.springerlink.com/content/q26131ut15503w15/

[9] T. H. Hong, H. L. Kim, Y. S. Lee, J. J. Kim, K. H. Lee, Y. K. You, S. J. Oh and S. M. Park, "Transumbillical Single-Port Laparoscopic Appendectomy,” Journal of Laparoendoscopic \& Advanced Surgical Techniques. Part A, Vol. 19, No. 1, February 2009, pp. 75-78. doi:10.1089/lap.2008.0338

[10] M. Desai, "Single-Port Surgery for Donor Nephrectomy: A New Era in Laparoscopic Surgery?” Nature Clinical Practice Urology, Vol. 6, No. 1, January 2009, p. 1. doi:10.1038/ncpuro1278

[11] J. H. Kaouk, R. K. Goel, G. P. Haber, S. Crouzet, M. M. Desai and I. S. Gill, "Single-Port Laparoscopic Radical Prostatectomy,” Urology, Vol. 72, No. 6, December 2008, pp. 1190-1193.

[12] J. Leroy, R. A. Cahil, M. Asakuma, B. Dallemagne and J. Marescaux, "Single-Access Laparoscopic Sigmoidectomy as Definitive Surgical Management of Prior Diverticulitis in a Human Patient," Archives of Surgery, Vol. 144, No. 2, February 2009, pp. 173-179. doi:10.1001/archsurg.2008.562
[13] J. Teixeira, K. McGill, S. Binenbaum and G. Forrester, "Laparoscopic Single-Site Surgery for Placement of an Adjustable Gastric Band: Initial Experience,” Surgical Endoscopy, Vol. 23, No. 6, June 2009, pp. 1409-1414. doi:10.1007/s00464-009-0411-9

[14] M. G. Neto, A. Ramos and J. Campos, "Single Port Laparoscopic Access Surgery,” Techniques in Gastrointestinal Endoscopy, Vol. 11, No. 2, April 2009, pp. 84-93. doi:10.1016/j.tgie.2009.06.007

[15] A. Moreno-Egea, J. A. Bustos, E. Girela and J. L. Aguayo-Albasini, "Long-Term Results of Laparoscopic Repair of Incisional Hernias Using an Intraperitoneal Composite Mesh,” Surgical Endoscopy, Vol. 24, No. 2, February 2010, pp. 359-365. doi:10.1007/s00464-009-0573-5

[16] S. Olmi, A. Scaini, L. Erba, A. Bertolini and E. Croce, "Laparoscopic Repair of Inguinal Hernias Using an Intraperitoneal Onlay Mesh Technique and a Parietex Composite Mesh Fixed with Fibrin Glue (Tissucol). Personal Technique and Preliminary Results," Surgical Endoscopy, Vol. 21, No. 11, November 2007, pp. 19611964. doi:10.1007/s00464-007-9355-0 